\title{
Magnetic clouds along the solar cycle: expansion and magnetic helicity
}

\author{
Sergio Dasso ${ }^{1,2}$, Pascal Démoulin ${ }^{3}$, and Adriana M. Gulisano ${ }^{1,4}$ \\ ${ }^{1}$ Instituto de Astronomía y Física del Espacio, CONICET-UBA, CC. 67, Suc. 28, 1428 Buenos \\ Aires, Argentina \\ email: dasso@df.uba.ar \\ ${ }^{2}$ Departamento de Física, Facultad de Ciencias Exactas y Naturales, Universidad de Buenos \\ Aires, 1428 Buenos Aires, Argentina \\ ${ }^{3}$ Observatoire de Paris, LESIA, UMR 8109 (CNRS), F-92195 Meudon Principal Cedex, France \\ ${ }^{4}$ Instituto Antártico Argentino (DNA), Cerrito 1248, Buenos Aires, Argentina
}

\begin{abstract}
Magnetic clouds (MCs) are objects of extreme importance in the heliosphere. They have a major role on releasing magnetic helicity from the Sun (with crucial consequences on the solar dynamo), they are the hugest transient object in the interplanetary medium, and the main actors for the Sun-Earth coupling. The comparison between models and observations is beginning to clarify several open questions on MCs, such as their internal magnetic configuration and their interaction with the ambient solar wind. Due to the decay of the solar wind pressure with the distance to the Sun, MCs are typically in expansion. However, their detailed and local expansion properties depend on their environment plasma properties. On the other hand, while it is well known that the solar cycle determines several properties of the heliosphere, the effects of the cycle on MC properties are not so well understood. In this work we review two major properties of MCs: (i) their expansion, and (ii) the magnetic flux and helicity that they transport through the interplanetary medium. We find that the amount of magnetic flux and helicity released via MCs during the last solar minimum (years 2007-2009) was significantly lower than in the previous one (years 1995-1997). Moreover, both MC size and mean velocity are in phase with the solar cycle while the expansion rate is weakly variable and has no relationship with the cycle.
\end{abstract}

Keywords. magnetic fields, magnetic clouds, coronal mass ejections (CMEs), solar wind, interplanetary medium, plasmas

\section{Introduction}

The study of the heliosphere has advanced greatly in the last few years. The comparison between models and observations is clarifying several issues of this system. The synergy of combining modeling with different observational techniques has led to a very important progress in our understanding of the heliosphere, with important consequences on SunEarth connection and space weather.

\subsection{Magnetic clouds}

Interplanetary Coronal Mass Ejections (ICMEs) are the biggest transient structures in the solar wind (SW). An important set of ICMEs is known as Magnetic Clouds (MCs), a term introduced by Burlaga et al. (1981). A MC is characterized by an enhanced magnetic field strength with respect to ambient values, a smooth and large rotation of the magnetic field vector, and low proton temperature (e.g., Burlaga et al. 1981; Klein \& Burlaga 1982; Burlaga 1995).

When a MC is traveling in the Earth direction, it can be geoeffective. In particular, depending on the orientation and strength of its magnetic field, a MC can initiate intense 

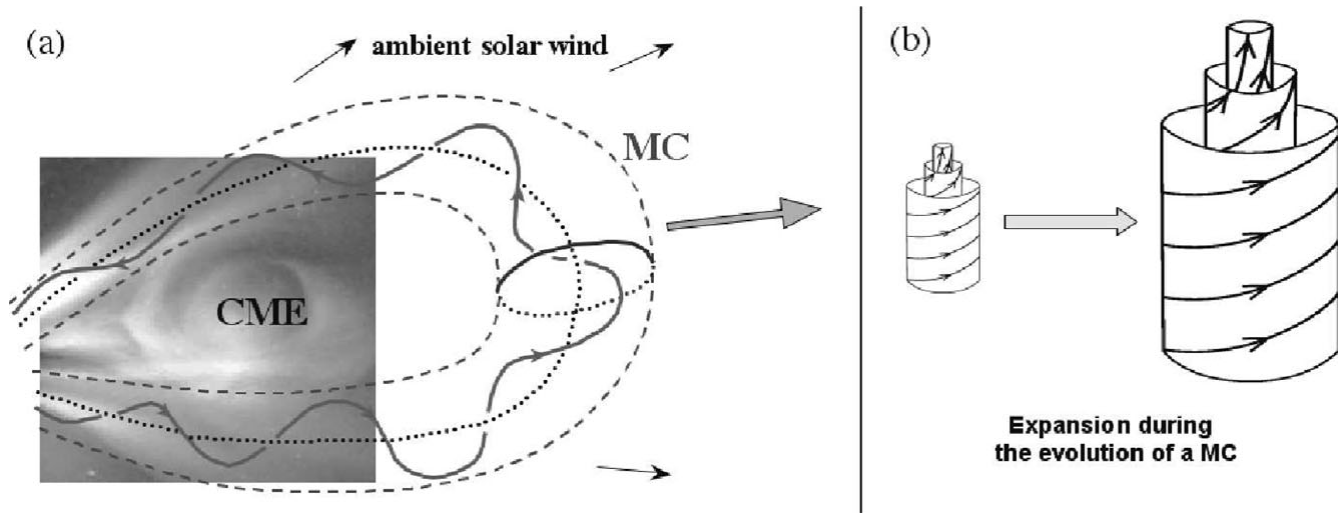

Figure 1. (a) Cartoon of a CME-MC interacting with the surrounding solar wind plasma while evolving in the interplanetary medium (the CME image is from SOHO/LASCO). (b) Due to the decrease of the solar wind pressure with the distance to the Sun, MCs are globally expanding objects.

geomagnetic perturbations as consequence of reconnection processes in the terrestrial magnetopause (e.g., Gonzalez et al. 1999, and references therein). It is worth noting that the field strength depends both on the solar source region and on the MC expansion during its transit from the Sun to Earth (because of the conservation of magnetic flux). Moreover, fast MCs generally drive a shock wave, which can increase the geoeffectiveness of the event.

MCs can be modeled locally using an helical cylindrical geometry as a first approximation (e.g., Farrugia et al. 1995). Several models have been used for describing the magnetic structure of the local cylinder of MCs. The most frequently used, is the linear force-free field (e.g., Burlaga et al. 1981; Burlaga 1988; Lepping et al. 2003), which corresponds to the first eigen-function of the curl operator for cylindrical symmetry.

A cylindrical non-linear force-free and uniformly twisted field (e.g., Farrugia et al. 1999) has been used as a possible alternative to describe MCs. Also several non forcefree models have been applied, using different shapes for their cross sections (e.g., Hu \& Sonnerup 2001; Cid et al. 2002; Hidalgo et al. 2002; Vandas \& Romashets 2003; Démoulin \& Dasso 2009b). All these models are physically different. Comparisons of these models with observations and synthetic data have been done (e.g., Riley et al. 2003; Dasso et al. $2005 \mathrm{~b})$ but it is not yet clear which of them gives the best representation of MCs.

MCs are evolving while traveling in the solar wind. Indeed, it is very frequent to observe an 'in situ' velocity profile of MCs with a clear signature of expansion (i.e. with a faster velocity in the front than at the rear, e.g. Gulisano et al. 2010). Figures 1a and 1b show a cartoon of a solar eruption, launching a flux rope, and the consequent expansion of the associated MC. Several models have considered the expansion effects on the decay of the observed magnetic profile (e.g., Shimazu \& Vandas 2002; Berdichevsky et al. 2003; Démoulin \& Dasso 2009a; Nakwacki et al. 2008, 2011).

The large scale magnetic structure of MCs is helical, then these objects transport large amounts of magnetic flux $(F)$ and helicity $(H)$ from the Sun to the outer heliosphere. The content of $F$ and $H$ in MCs have been quantified using several models (e.g., Dasso et al. 2005b; Lynch et al. 2005). Nakwacki et al. (2008) have estimated the bias produced by the MC expansion on $F$ and $H$. By comparing static and expanding models, they have concluded that these quantities do not differ more than $25 \%$ when using both kind of approaches. 


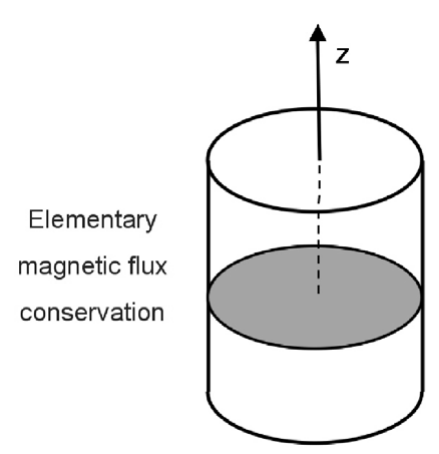

$\mathrm{FZ}_{\mathbf{Z}}$

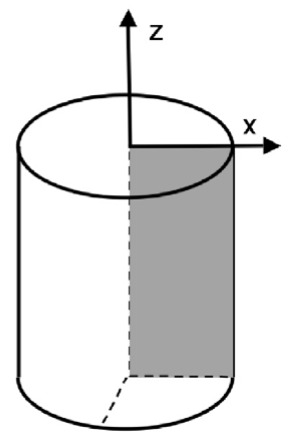

Fy

Figure 2. Scheme of surfaces associated with $F_{z}$ (axial flux) and $F_{y}$ (azimuthal flux).

The twisted magnetic structures associated with a large amount of $H$ transported by MCs have important consequences also on the propagation of energetic particles in the solar wind. In particuliar, Masson et al. (2012) have analyzed the Earth-arrival time for high energy particle released from the Sun during relativistic solar particle events. They have shown that this Earth-arrival time is different when the interplanetary medium presents structures as the Parker's model for the SW and when it presents transient structures as ICMEs/MCs.

\subsection{Eruptive events along the solar cycle}

During the last solar maximum (solar cycle 23) an important number of large active regions with long lifetimes was observed. These ARs produced several huge magnetic clouds, which transported very large amounts of magnetic flux and helicity into the heliosphere. Some of the magnetic clouds launched during this period were associated with a complex topology of the coronal magnetic field (e.g. Schmieder et al. 2011).

On the other hand, the last solar minimum (minimum of solar cycle 24) presented the slowest and least dense solar wind, and the weakest interplanetary magnetic field, when compared with the last three minima periods (Jian et al. 2011). Coronal holes near the ecliptic produced recurrent fast streams of fast solar wind during this last minima (de Toma 2011). There was also an north-south asymmetry of the AR locations, with more ARs located in the South (de Toma 2012, this volume). This implies an expected predominance of positive helicity for ICMEs which erupted in this period. A comparison of a variety of outward-traveling transients observed in the solar corona during these two last solar minima, as achieved during the observing campaigns of the Whole Sun Month (WSM, from 10 August to 8 September 1996) and of the Whole Heliosphere Interval (WHI, from 20 March to 16 April 2008) was made by Cremades et al. (2011). They found more ejecta produced by active regions during WHI than during WSM.

\section{Global magnetic quantities}

\subsection{Magnetic field and cross section}

MCs have low plasma $\beta$ (e.g., Lepping et al. 2003), they present a configuration in a quasi-equilibrium (a state near to a force-free field), and they are formed by a flux rope magnetic structure. Then, the magnetic forces dominate the dynamics inside the structure of MCs for a given time and their magnetic configuration can be modeled using the cylindrical linear force-free field $\overrightarrow{B^{L}}=B_{0}\left[J_{1}\left(\alpha_{0} r\right) \vec{\phi}+J_{0}\left(\alpha_{0} r\right) \vec{z}\right]$ (Lundquist 1950). 
There are evidences both from MHD simulations (Riley et al. 2003; Manchester et al. 2004) and from observations (Owens et al. 2006; Savani et al. 2011; Nakwacki et al. 2011) that MCs propagating in the interplanetary medium have a trend to develop oblate shapes for their cross sections. This effect is mainly due to the interaction between the MC and the solar wind (Vandas et al. 1995; Riley et al. 2003). However, the aspect ratio between the major axis (which is expected to be perpendicular to the Sun-ward direction) with respect to the minor one seems to be moderate (typically $\leqslant 2$ ). In particular, from a reconstruction of the magnetic cross section using a Grad-shafranov formalism, Liu et al. (2008) found that the MC core ( the inner half part of the flux rope) is almost circular, while the flux rope periphery is more oblate [see Figure 5 of Liu et al. 2008]. This result can be interpreted as a consequence of magnetic tension, which will favor a circular shape when the magnetic field is strong enough. The deformation of the external part can be interpreted as a consequence of the interaction with the ambient solar wind.

\subsection{Magnetic flux and helicity}

Magnetic flux $(F)$ and helicity $(H)$ are ideal magnetohydrodynamical (MHD) invariants. Magnetic helicity quantifies the twist and linkage of magnetic field lines and it is approximately conserved in the solar atmosphere and the heliosphere because of the high magnetic Reynolds number (Berger 1984). Since MCs are large twisted flux tubes, they transport important amounts of $H$ through the solar wind (a review on magnetic helicity in MCs can be found in Dasso 2009).

A quantification of $F$ and $H$ can be done using a specific model for describing the MC magnetic configuration. A local frame of coordinates linked to the cloud is very useful to make quantitative comparisons between models and observations, and to quantify $F$ and $H$.

Figure 2 shows a cartoon showing the local system (for a precise definition of this system, see e.g., Dasso et al. 2005b). The magnetic fluxes are computed across two surfaces: across a surface perpendicular to $\hat{z}\left(F_{z}\right.$, the axial flux) and across a surface perpendicular to $\hat{y}\left(F_{y}\right.$, the azimuthal flux). Simple analytical expressions for $F_{z}, F_{y}$, and $\mathrm{H}$, can be derived in function of the free parameters of the Lundquist model $\left(\alpha_{0}, B_{0}\right)$ and the radius of the cross section $(R)$ (e.g., Dasso et al. 2005b) and thus, these quantities can be evaluated after fitting these parameters to observations of a given cloud.

Different methods and models, to quantify $F$ and $H$ from in situ observations, have also been used (e.g., Dasso et al. 2005b; Lynch et al. 2005; Nakwacki et al. 2008, 2011). Comparison of estimations for $F$ and $H$ obtained from different models, that fit relatively well to in situ observations of several samples of MCs, have been done (e.g., Gulisano et al. 2005; Dasso et al. 2005a; Nakwacki et al. 2008). These studies concluded that, for a given event, differences on estimations of $F$ and $H$ using different models are typically much lower than the dispersion of these quantities for different events. These results imply that helicity and fluxes are relatively well defined quantities for MCs.

Quantitative studies of $F$ and $H$ both in MCs and in the solar corona are used to relate MCs to their solar sources (using $F$ and $H$ approximative conserved properties). Moreover, these studies set constrains to coronal magnetic configurations and on flux rope formation/eruption models (Mandrini et al. 2005; Luoni et al. 2005; Attrill et al. 2006; Longcope et al. 2007; Qiu et al. 2007; Harra et al. 2007; Mandrini et al. 2007; Rodriguez et al. 2008; Möstl et al. 2008; Ravindra et al. 2011).

\subsection{Magnetic flux and helicity in MCs during the last two minima}

Lepping et al. (2011) studied some properties of MCs observed at one astronomical unit by the spacecraft Wind in the two last solar minima. In particular they study MCs during 

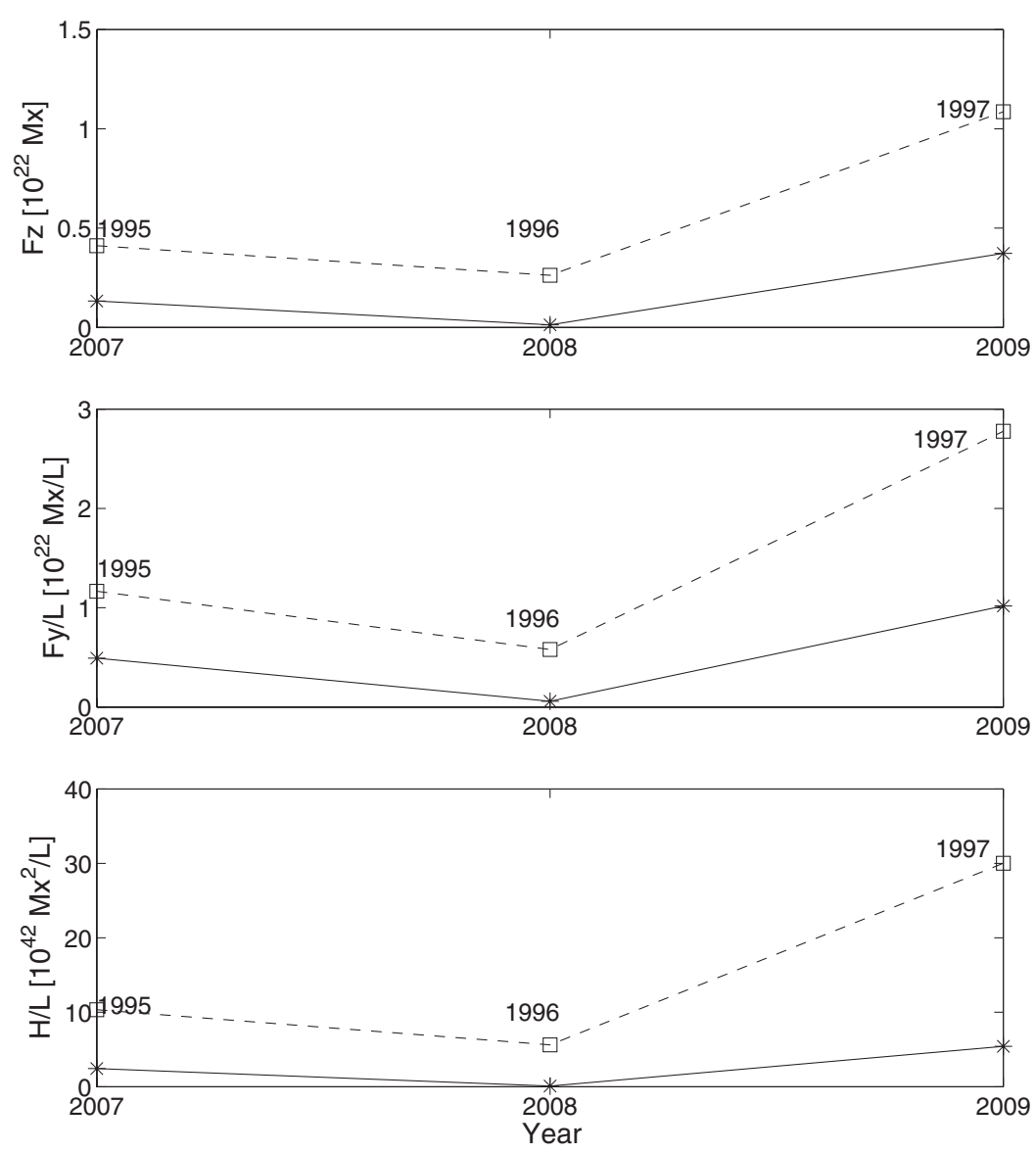

Figure 3. Comparison of magnetic quantities in MCs cumulated in each year of periods I and II (see Section 2.3). From upper to lower panels: axial flux $\left(F_{z}\right)$, azimuthal flux per unit length $\left(F_{y} / L\right)$, and magnetic helicity per unit length $(H / L)$. All these magnetic quantities are lower in Period II than in period I.

the previous solar minimum (years 1995-1997, which we call period I) and during the last recent minimum (year 2007-2009, which we call period II). From a comparison of periods I and II, they find that period II presented: (1) a lower number of events (29 MCs for period I and 18 MCs for period II), (2) a significantly lower axial magnetic field $\left(B_{0}\right.$ is in average lower by $\left.33 \%\right)$, (3) similar velocities, (4) lower duration of the 'in situ' observations, then (5) smaller MC size, and $(6) \sim 50 \%$ less axial flux $\left(F_{z}\right)$, than during period I.

In our study we analyze the same set of MCs studied by Lepping et al. (2011), and from using the fitted free parameters of the Lundquist's model $\left(B_{0}, \alpha_{0}\right.$, and $R$, see Section 2.2), we compute the axial $\left(F_{z}\right)$ and the azimuthal $\left(F_{y}\right)$ fluxes, and the magnetic helicity $(H)$. The results are shown in Figure 3. This figure follows the style used in Figure 1 of Lepping et al. (2011). In that paper the authors show the number of events in the two periods, we show here the cumulated magnetic quantities considering all the MCs observed in each year.

Figure 3 shows that, during each of the years of period II, MCs transported a significantly lower amount of magnetic flux and helicity, when compared with the corresponding years in period I. The fraction of total content of fluxes and helicity, considering all the 

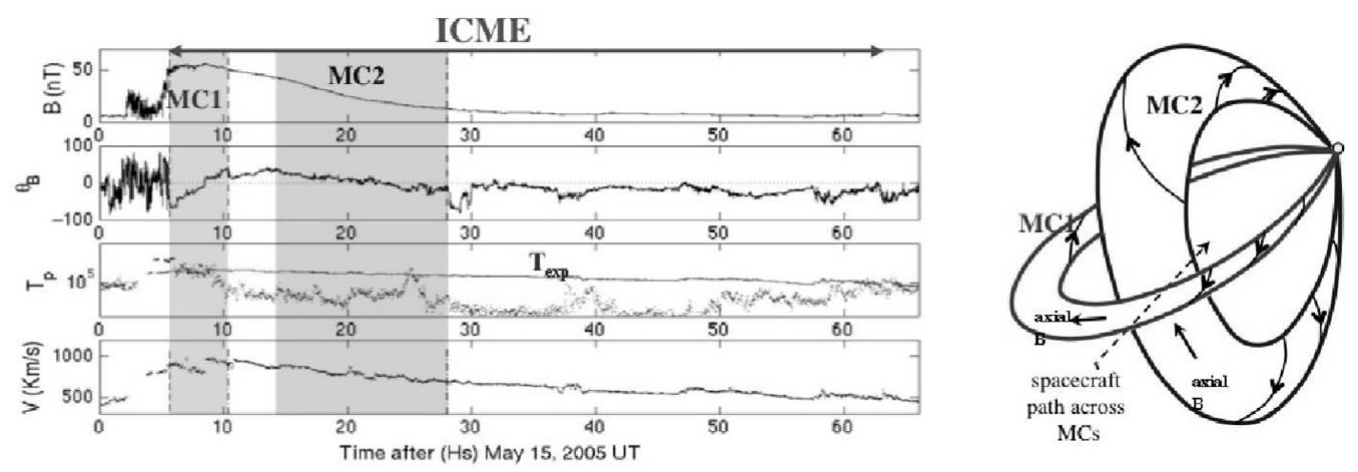

Figure 4. The interaction between two magnetic clouds affect their expansion rate. This figure shows 'in situ' observations (left) and a cartoon of two interacting MCs (right), observed in May 2005. MC1 is strongly compressed by the overtaking MC2, and the velocity profile of MC1 shows almost no expansion, while MC2 has a classical expansion profile (Dasso et al. 2009).

events for each period, is: $F_{z}^{I I} / F_{z}^{I} \sim 0.29, F_{y}^{I I} / F_{y}^{I} \sim 0.35, H^{I I} / H^{I} \sim 0.17$. Moreover, a typical MC in period II transported less flux and helicity than a typical MC in period I. The mean (median) values of $F$ and $H$ ratios for MCs in period II with respect to period I are: $\left\langle F_{z}^{I I}\right\rangle /<F_{z}^{I}>\sim 0.47(0.35),\left\langle F_{y}^{I I}\right\rangle /<F_{y}^{I}>\sim 0.56(0.51)$, $<H^{I I}>/<H^{I}>\sim 0.28(0.15)$. Then, a lower total magnetic flux and helicity released from the Sun in period II as MCs comes from a combination of (i) less number of events and (ii) weaker MCs during period II with respect to period I.

\section{Expansion}

\subsection{Self-Similar expansion}

The expansion of MCs can be quantified from the 'in situ' observations of the proton velocity profile. The expansion can be described using a model developed by Démoulin et al. (2008), which is derived from a few basic hypothesis:

(1) The motion of parcels of fluid inside a MC can be split in two: (a) a global motion describing the position $\vec{r}_{C M}(t)=D(t) \hat{\mathbf{v}}_{C M}$ of the center of mass (CM) with respect to a fixed heliospheric frame and (b) an internal expansion where the fluid elements are expressed relative to the $\mathrm{CM}$.

(2) During the MC crossing the spacecraft, the MC center travels with an almost uniform speed $\left(\vec{v}_{C M}\right.$, with $\left.\left|\vec{v}_{C M}\right|=V_{0}\right)$, then

$$
D(t)=D_{0}+V_{0}\left(t-t_{0}\right)
$$

(3) The local MC frame $(\hat{\mathbf{x}}, \hat{\mathbf{y}}, \hat{\mathbf{z}})$ (see Figure 2), defines the three principal directions of expansion.

(4) The flux rope expansion is self similar with different expansion rates in each of the three principal directions.

These assumptions imply that the position at time $t$ of an element of fluid is described as:

$$
\begin{aligned}
\vec{r}(t) & =x(t) \hat{\mathbf{x}}+y(t) \hat{\mathbf{y}}+z(t) \hat{\mathbf{z}} \\
& =x_{0} e(t) \hat{\mathbf{x}}+y_{0} f(t) \hat{\mathbf{y}}+z_{0} g(t) \hat{\mathbf{z}},
\end{aligned}
$$

with $x(t), y(t), z(t)$ being the fluid coordinates in the local frame, and $x_{0}, y_{0}, z_{0}$ the 

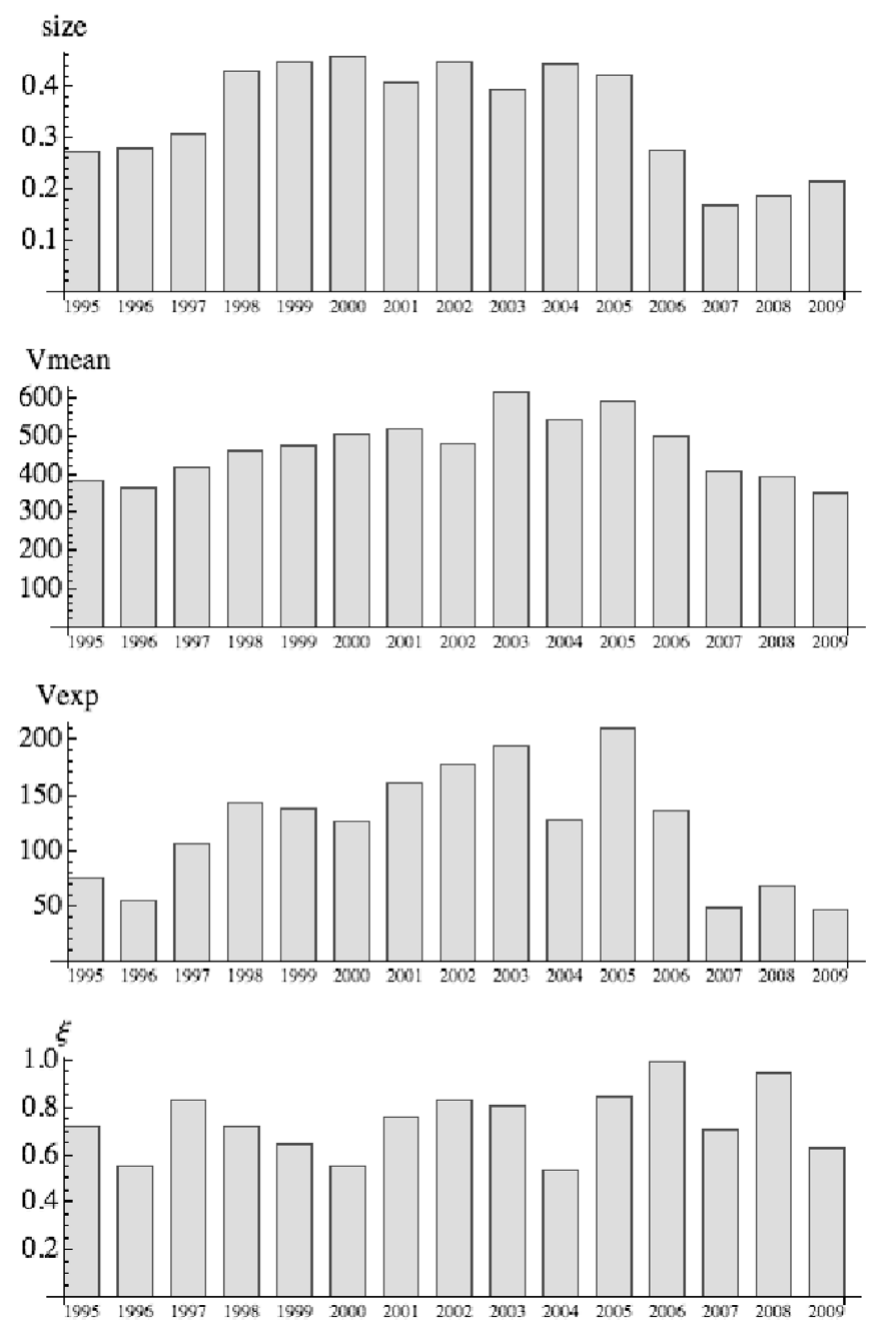

Figure 5. Distribution of the MC properties along the solar cycle. From the top to the bottom panels: size, mean velocity, expansion velocity, and expansion rate $(\zeta)$.

reference positions at time $t_{0}$. The functions $e(t), f(t)$, and $g(t)$, provide the specific time dependences for the self-similar evolution.

Based on observations of MCs at different distance from the Sun (e.g., Liu et al. 2005; Wang et al. 2005; Leitner et al. 2007; Gulisano et al. 2010), it is possible to approximate $e(t)$ as:

$$
e(t)=\left(D(t) / D_{0}\right)^{l}
$$

Similar expressions can be used for $f(t)$ and $g(t)$ (replacing the exponent $l$ by $m$ and $n$, respectively).

Neglecting the evolution of the spacecraft position while it observes the MC, the measured velocity profile $\left(V_{x}\right)$ along the direction $\hat{\mathbf{v}}_{C M}$ is expected to be (Démoulin et al. 2008):

$$
V_{x}=-V_{0}+V_{0} \frac{t-t_{0}}{D_{0} / V_{0}+t-t_{0}} \zeta
$$




$$
\begin{aligned}
& \approx-V_{0}+\frac{V_{0}^{2}}{D_{0}} \zeta\left(t-t_{0}\right), \\
\text { with } \quad \zeta & =l \sin ^{2} \gamma+n \cos ^{2} \gamma,
\end{aligned}
$$

and with the angle between $\hat{\mathbf{z}}_{\text {cloud }}$ and $\hat{\mathbf{v}}_{C M}$ defined as $\gamma$. Thus, with an isotropic expansion in two directions $(l=n)$, the slope of the observed linear velocity profile allows us to determine the expansion rate, $\zeta$, of the flux rope (for anisotropic expansion and acceleration effects on the velocity profile see Démoulin et al. 2008; Nakwacki et al. 2011).

\subsection{Expansion along the cycle}

We analyze a set of 75 magnetic clouds observed by the spacecraft ACE at one astronomical unit from 1995 to 2009 . For each event, we quantify the mean velocity $\left(V_{\text {mean }}\right)$, the size $(S)$, the expansion velocity $\left(V_{\text {exp }}\right)$, and the expansion rate $(\zeta) . S$ is derived from the mean velocity and the MC duration, while $V_{\text {exp }}$ and $\zeta$ are both derived from a linear fit of the observed velocity profile. Figure 5 shows the mean value of these quantities inside bins of one year. We found that $S, V_{\text {mean }}$ and $V_{\text {exp }}$ are in phase with the solar cycle (in agreement with Jian et al. 2011), with larger and faster MCs near the solar maximum, and smaller and slower MCs observed in periods of solar minimum.

The expansion rate, $\zeta$, has only weak variations and it does not follow the solar cycle. The dispersion of $\zeta$ from its theoretically expected value of $\sim 0.7-0.9$ (Démoulin \& Dasso 2009a) can be interpreted as a consequence of the perturbation of the observed cloud by fast solar wind streams, or by another MC (Gulisano et al. 2010). Thus, this result is in agreement with MCs which can be perturbed during all the phases of the solar cycle.

Moreover, for MCs at a distance $D$ from the Sun there is a relationship between $\zeta$ and $V_{\text {exp }}$ given by $V_{\text {exp }}=\zeta V_{\text {mean }} S / D$. Then, from the non-dependence of $\zeta$ with the solar cycle (lower panel of Figure 5) and from the increasing behaviour of $V_{\text {mean }}$ and $S$ with solar activity, a strong dependence of $V_{\text {exp }}$ with the cycle is expected, as indeed present (third panel of Figure 5).

The above results show that MCs properties, apart from the expansion rate $\zeta$, are cycle dependant. Such dependence needs to be taken into account when a typical MC is used, for example for building a magnetic helicity budget (e.g. Démoulin 2007, and references therein).

\section{Summary and Conclusions}

In this work we revised methods which quantify the content of magnetic flux and helicity in MCs from 'in situ' observations of the magnetic field, and also methods which quantify their expansion from 'in situ' observations of the proton velocity. We also studied the influence of the solar cycle on these MC properties.

We compared the amount of flux and helicity released from the Sun during the last two minima and found that, due to a less number of events and less flux and helicity contained in each typical event, the amount of $H(F)$ released via MCs during the last solar minimum (years 2007-2009) was lower than 20\% (35\%) than during the previous one (years 1995-1997). MCs are one of the main agents in charge of releasing magnetic helicity from the Sun. Then, these quantitative results can be useful for better understanding the solar dynamo and then to improve our knowledge of the so weak last solar minimum itself.

We studied also the variability of the MCs expansion along the solar cycle, and found that the dimensionless expansion parameter $(\zeta)$ has no dependence with the solar cycle. 
This results is coherent with previous ones because $\zeta$ mainly depends on the global decay of the solar wind pressure with the distance to the Sun (Démoulin \& Dasso 2009a). However, because of the dependence of both the size and speed of MCs with the solar magnetic activity, the velocity of expansion $\left(V_{\text {exp }}\right)$ has a strong dependence with the solar cycle (with a significantly lower expansion during solar minima). Moreover, as a consequence of MC interaction with streams of the fast solar wind during periods of solar minima (as occurred in the last minimum), MCs can be perturbed even during periods with low level of solar activity.

\section{Acknowledgments}

The authors acknowledge financial support from ECOS-Sud through their cooperative science program ( $\mathrm{N}^{\circ}$ A08U01). S.D. is a member of the Carrera del Investigador Cientifico, CONICET. S.D. and A.M.G. acknowledge partial support by Argentinean grants UBACyT 20020090100264 (UBA), PIP 11220090100825/10 (CONICET), PICT 2007856 (ANCPyT). S.D. acknowledges support from the Abdus Salam International Centre for Theoretical Physics (ICTP), as provided in the frame of his regular associateship.

\section{References}

Attrill, G., Nakwacki, M. S., Harra, L. K., et al. 2006, Solar Phys., 238, 117

Berdichevsky, D. B., Lepping, R. P., \& Farrugia, C. J. 2003, Phys. Rev. E, 67, 036405

Berger, M. A. 1984, Geophys. Astrophys. Fluid Dyn., 30, 79

Burlaga, L., Sittler, E., Mariani, F., \& Schwenn, R. 1981, J. Geophys. Res., 86, 6673

Burlaga, L. F. 1988, J. Geophys. Res., 93, 7217

Burlaga, L. F. 1995, Interplanetary magnetohydrodynamics (Oxford University Press, New York)

Cid, C., Hidalgo, M. A., Nieves-Chinchilla, T., Sequeiros, J., \& Viñas, A. F. 2002, Solar Phys., 207,187

Cremades, H., Mandrini, C. H., \& Dasso, S. 2011, Solar Phys., 274, 233

Dasso, S. 2009, in IAU Symposium, Vol. 257, IAU Symposium, ed. N. Gopalswamy \& D. F. Webb, pp. 379-389

Dasso, S., Gulisano, A. M., Mandrini, C. H., \& Démoulin, P. 2005a, Advances in Space Research, 35,2172

Dasso, S., Mandrini, C. H., Démoulin, P., Luoni, M. L., \& Gulisano, A. M. 2005b, Adv. Spa. Res., 35, 711

Dasso, S., Nakwacki, M., Démoulin, P., \& Mandrini, C. H. 2007, Solar Phys., 244, 115

Dasso, S., Mandrini, C. H., Schmieder, B., et al. 2009, J. Geophys. Res., 114, A02109

de Toma, G. 2011, Solar Phys., 274, 195

de Toma, G. 2012, This volume

Démoulin, P. 2007, Adv. Spa. Res., 39, 11, 1674

Démoulin, P. \& Dasso, S. 2009a, Astron. Astrophys., 498, 551

Démoulin, P. \& Dasso, S. 2009b, Astron. Astrophys., 507, 969

Démoulin, P., Nakwacki, M. S., Dasso, S., \& Mandrini, C. H. 2008, Solar Phys., 250, 347

Farrugia, C. J., Janoo, L. A., Torbert, R. B., et al. 1999, in Habbal, S. R., Esser, R., Hollweg, J. V., Isenberg, P. A. (eds.), Solar Wind Nine, AIP Conf. Proc., Vol. 471, 745

Farrugia, C. J., Osherovich, V. A., \& Burlaga, L. F. 1995, J. Geophys. Res., 100, 12293

Gonzalez, W. D., Tsurutani, B. T., \& Clúa de Gonzalez, A. L. 1999, Space Sci. Rev., 88, 529

Gulisano, A. M., Dasso, S., Mandrini, C. H., \& Démoulin, P. 2005, JASTP, 67, 1761

Gulisano, A. M., Démoulin, P., Dasso, S., Ruiz, M. E., \& Marsch, E. 2010, Astron. Astrophys., 509, A39

Harra, L. K., Crooker, N. U., Mandrini, C. H., et al. 2007, Solar Phys., 244, 95

Hidalgo, M. A., Cid, C., Vinas, A. F., \& Sequeiros, J. 2002, J. Geophys. Res., 107, 1002

Hu, Q. \& Sonnerup, B. U. Ö. 2001, Geophys. Res. Lett., 28, 467 
Jian, L. K., Russell, C. T., \& Luhmann, J. G. 2011, Solar Phys., 274, 321

Klein, L. W. \& Burlaga, L. F. 1982, J. Geophys. Res., 87, 613

Leitner, M., Farrugia, C. J., Möstl, C., et al. 2007, J. Geophys. Res., 112, A06113

Lepping, R. P., Berdichevsky, D. B., Szabo, A., Arqueros, C., \& Lazarus, A. J. 2003, Solar Phys., 212, 425

Lepping, R. P., Wu, C.-C., Berdichevsky, D. B., \& Szabo, A. 2011, Solar Phys., 274, 345

Liu, Y., Luhmann, J. G., Huttunen, K. E. J., et al. 2008, Astrophys. J. Lett., 677, L133

Liu, Y., Richardson, J. D., \& Belcher, J. W. 2005, Planetary Spa. Sci., 53, 3

Longcope, D., Beveridge, C., Qiu, J., et al. 2007, Solar Phys., 244, 45

Lundquist, S. 1950, Ark. Fys., 2, 361

Luoni, M. L., Mandrini, C. H., Dasso, S., van Driel-Gesztelyi, L., \& Démoulin, P. 2005, JASTP 67,1734

Lynch, B. J., Gruesbeck, J. R., Zurbuchen, T. H., \& Antiochos, S. K. 2005, J. Geophys. Res., 110, A08107

Manchester, W. B. I., Gombosi, T. I., Roussev, I., et al. 2004, J. Geophys. Res., 109, A02107

Mandrini, C. H., Nakwacki, M., Attrill, G., et al. 2007, Solar Phys., 244, 25

Mandrini, C. H., Pohjolainen, S., Dasso, S., et al. 2005, Astron. Astrophys., 434, 725

Masson, S., Démoulin, P., Dasso, S., \& Klein, K.-L. 2012, Astron. Astrophys., 538, A32

Möstl, C., Miklenic, C., Farrugia, C. J., et al. 2008, Annales Geophysicae, 26, 3139

Nakwacki, M., Dasso, S., Démoulin, P., Mandrini, C. H., \& Gulisano, A. M. 2011, Astron. Astrophys., 535, A52

Nakwacki, M., Dasso, S., Mandrini, C. H., \& Démoulin, P. 2008, JASTP, 70, 1318

Owens, M. J., Merkin, V. G., \& Riley, P. 2006, J. Geophys. Res., 111, 3104

Qiu, J., Hu, Q., Howard, T. A., \& Yurchyshyn, V. B. 2007, Astrophys. J., 659, 758

Ravindra, B., Yoshimura, K., \& Dasso, S. 2011, Astrophys. J., 743, 33

Riley, P., Linker, J. A., Mikić, Z., et al. 2003, J. Geophys. Res., 108, 1272

Rodriguez, L., Zhukov, A. N., Dasso, S., et al. 2008, Annales Geophysicae, 26, 213

Savani, N. P., Owens, M. J., Rouillard, A. P., et al. 2011, Astrophys. J., 732, 117

Schmieder, B., Démoulin, P., Pariat, E., et al. 2011, Adv. Spa. Res., 47, 2081

Shimazu, H. \& Vandas, M. 2002, Earth, Planets, and Space, 54, 783

Vandas, M., Fischer, S., Dryer, M., Smith, Z., \& Detman, T. 1995, J. Geophys. Res., 100, 12285

Vandas, M. \& Romashets, E. P. 2003, Astron. Astrophys., 398, 801

Wang, C., Du, D., \& Richardson, J. D. 2005, J. Geophys. Res., 110, A10107

\section{Discussion}

Axel Brandenburg: Are there different signs of the helicity? In which hemisphere did you see positive helicity? Is the sign of the helicity of the ICME the same as in the active region?

Sergio DAsso: Yes, there are magnetic clouds with positive global helicity (righthanded) and there are others with negative one (left-handed). Active regions with positive helicity are typically observed in the southern solar hemisphere. The sign of the helicity of the clouds is the same as in the source active regions (e.g. Mandrini et al. 2005; Dasso et al. 2007; Longcope et al. 2007; Dasso et al. 2009; Nakwacki et al. 2011), with just a few exceptions in complex regions (e.g. Schmieder et al. 2011). In fact, the last minimum was more active in the southern hemisphere and, consequently, there were more clouds with positive helicity. 